\title{
The neural effects of alcohol
}

\author{
Clairmont Griffith and Bernice La France* \\ Howard University Hospital and Howard University College of Medicine
}

\begin{abstract}
This paper focuses on the harmful cognitive and neurotoxic effects of alcohol. The paper will study journals to establish evidence of cognitive and neurotoxic effects of alcohol on its users. Alcoholism is as a major cause of diseases, injuries, and death. Studies have established that alcoholics are at a higher risk of developing severe and persistent changes in their brain. The brain of adolescents is vulnerable to alcohol hence the restrictions on underage drinking. Damage to the brain interferes with brain-controlled functions. The effects of brain damage due to alcohol consumption might result in the loss of sight, memory loss, impaired motor functions, slowed reaction and death. The effect of alcohol has become a subject of interest with the worst effect being its effect on the nervous system. The effects of, alcohol on the central nervous system can manifest after a few drinks or after prolonged indulgence in alcohol. Prolonged indulgence in alcohol might lead to brain deficits that persist for a longer period even after the individual abstains from alcohol. There are ongoing studies to establish how alcohol affects the brain and how to reverse these effects.
\end{abstract}

\section{Introduction}

Alcohol is a socially accepted intoxicating liquid substance produced by fermentation of sugars. Ethanol is the main alcoholic component found in beers, wines, and spirits. The chemical composition of ethanol and its weight makes it easy to diffuse into the blood system and into the central nervous system to give the partaker the feeling of being drunk. Alcohol has become the most abused substance globally since there is limited control over the quantity of alcohol an individual is allowed to consume and it is socially acceptable to get drunk. Addiction is also prevalent among those who use alcohol. Underage drinking is also an issue of concern. The neurotoxic effects of alcohol vary from mild to severe cases of brain damage. Alcohol has various mild and severe effects on the central nervous system. These effects alter an individual's behavior and biological functions [1]. In severe cases, the affected individual might develop irreversible or life-threatening conditions.

Drunk driving is also an issue of concern worldwide. It has been established that drunk drivers have impaired judgment and often endanger their lives and that of other road users. Impaired judgment is an altered function of the brain. The WHO has established alcoholism as a global burden. Alcoholics are likely to suffer from mental abnormalities and behavioral changes associated with impaired functions of their brain. Alcohol can affect the brain directly due to its toxicity or damage other body organs which affect the functioning of the brain nerve cells. Prolonged alcohol consumption has been scientifically proven to affect the structures of the brain. CT scans and magnetic resonance images of an alcoholic indicate change tissue damage and shrinking of the brain [2]. The result of such changes might be catastrophic to the individual.

\section{Method}

This paper examines published articles on the effects of alcohol on the neural effects of alcohol. The articles selected for this study were considered for their information based on practical research. The target population is the American population. The most important aspect that was considered in selecting appropriate literature for this study was the relevance of the topic on the specific effects of alcohol.
The articles used also were of the latest studies carried out within the last five years. The literature used for this paper studied both adults and underage alcoholics. The objective of this literature review is to establish the effects of alcohol on the central nervous system and the brain. The studies met all ethical research procedures.

\section{Results}

Sinha, [3] established that brain imaging produced positive results for brain-related changes as a result of chronic alcoholism. The study revealed stress, emotional and motivation and a consequence of alcohol-induced changes in the brain. The commonly affected regions of the brain include the ventral and dorsal striatum, the frontal and insular cortices, the amygdala, hippocampus, and thalamic nuclei, and midbrain regions [3]. Excessive abuse of alcohol and acute withdrawal heightens brain activity and has a direct effect on the stress systems. As a result, the brain increases the release of stress hormones corticotrophin-releasing factor (CRF) Cortisol and norepinephrine. The release of these stress hormones result in emotional changes and increase stress levels in alcoholics [3].

Alcohol is a proven psychotropic depressant of the central nervous system. It is known for inhibiting the functions of the central nervous system. When taken, alcohol enhances the production of neurosteroids which induce relaxation and inhibitory neurotransmitters. From their study, Costardi, et al. [1] established that when alcohol is taken over prolonged period damage the hippocampus. The damage to the hippocampal mass is responsible for memory loss in most alcoholics. The hippocampus part of the brain is responsible for

*Correspondence to: Bernice La France, Howard University Hospital and Howard University College of Medicine, 2041 Georgia Ave. NW, Washington, D.C. 20059, Tel: (240) 565-3358; E-mail: bemore1576@gmail.com

Key words: Alcohol, alcoholism, addiction indulgence, central nervous system, brain, heavy drinking

Received: September 26, 2018; Accepted: October 05, 2018; Published: October 09,2018 
memories that every human has. Alcohol reduces the hippocampal mass which is mediated by the GABA. This mass is responsible for explicit memories in humans where the individual is capable of making a clear recollection of immediate and long-term memories. The damage caused by alcohol to the hippocampus prevents an individual from recollecting recent/new memories. However, the individual retains the long-term memories [1].

\section{Discussion}

Research evidence indicates that prolonged and regular indulgence in alcohol leads to emotional changes and stress. Chronic alcoholism also interferes with the individual's sleep patterns, negative emotions, affects their appetite, changes their concentration, memory and increases their desire to drink. Welch, [4] found out that brain damage due to alcohol was high in individuals aged between 40 and 50 years old. Irreversible damages were also high among malnourished alcoholics who were heavy drinkers [4]. When a chronic alcoholic attempts to stop drinking, the stress level increase. Such stress is also manifested in users of other hard drugs. At this level of reaction, the use of alcohol becomes toxic to one's body and brain. Addiction sets in to the extent that the individual can barely function without alcohol. The individual's brain is at this point affected and conditions the body to a craving for alcohol. During withdrawal, the brain stress system becomes active. The individual's brain becomes active in secreting stress hormones and suppresses dopamine receptors. These studies identified altered brain response. Malfunctioning of the brain as a result of alcoholism is an indication of how severe the consequence of addiction can be. Once the brain's function is altered, a series of other complications develop. The brain of an intoxicated individual is unable to respond to the body's stress (Figure 1).

Another consequence is the malfunctioning sympathetic and parasympathetic components. Due to initiated stress activity by alcohol, the HPA axis is stimulated and arouses the sympathetic components. The heart rate variability also decreases in an alcohol intoxicated person. The brain of an intoxicated individual is at the risk of deregulating the neurochemical system. The neurochemical system plays an important role in the emotional and stress functions of the body. Therefore, the irregular functioning of the neurochemical system is the main cause of increased stress among alcoholics. This is mainly manifested in addicted individuals or a hangover after the alcohol intoxication subsides. In most occasions, individuals who experience such changes will complain of chronic headaches, be emotionally unstable, or have the desire to drink again to suppress the stress levels. Addiction is likely to set in during this phase [5]. Studies indicate that addicted individuals have more craving for alcohol and are likely to abuse it than social drinkers.

Alcoholism has been found to interfere with neurological processes such as sleep, body temperature, and coordination. Partaking alcohol lowers the body temperature according to the amount of alcohol consumed. Heavy drinking might lower body temperature to lifethreatening levels. The hypothalamus controls many body functions including learning and memory, and regulation of hormones, emotions, eating/drinking and the body temperature. Alcohol affects the electrophysiological activity of the neurons in the anterior hypothalamus which in turn affect the thermogenesis mechanisms.

\section{How alcohol affects the brain}

Cerebral Cortex: This is the main area

involved in thinking, decision-making, emotions, and the five senses. Alcohol's effects on this area can impair your ability to think clearly and lower your inhibitions. It may make you act without thinking or make you angry for no reason. Alcohol may affect your senses, such as blurring your vision. Long-term Drinking alcohol affects the way your
brain works - changing everything from the way you act to your ability to walk. Some effects can be long-lasting. Learn about how alcohol affects different parts of the brain. ${ }^{1}$

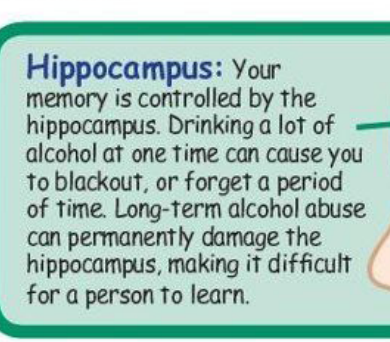

Hypothalamus: Many body processes, such as heart rate and the feeling of hunger or thirst, are controlled in this small area. Alcohol can slow your heart rate and may make you hungrier and thirstier.

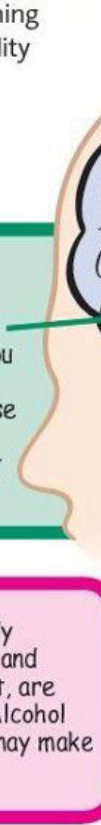

Figure 1. Pictorial summary off the effect of alcohol on the brain

Cerebellum: This part of the brain is important for coordinating many of your daily movements, such as walking and grabbing objects. Alcohol can slow your reflexes. It may cause you to lose your balance or make your hands shake.

Medulla: Involuntary processes, such as breathing and maintaining body temperature, are controlled here. Drinking a lot of alcohol at one time can shut down the medulla, leading to a coma.
Alcohol slows down this system. spinal cord, and nerves. That

affects how signals flow through your body, making you think, speak and move more slowly.

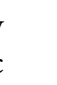 . .

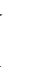

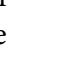


Consequently, overindulgence in alcohol leads to increased heat loss and slowed thermogenesis [6]. The notion among many alcoholics that drinking during cold weather will warm their bodies is dangerous. Alcohol also sedates an intoxicated individual into sleeping. However, while sleeping, the individual has little rapid-eye-movement (REM). REM is a critical neurological function when asleep [7]. Therefore, those who use alcohol as a sleeping sedative experience sleep fragmentation as the alcohol levels in their bodies is eliminated. Dependence on alcohol as a sleep sedative has negative effects since after withdrawal the individual might develop rapid REM and insomnia [8].

The cerebellum part of the brain is also harmed by chronic alcoholism. The cerebellum is a critical part of the brain whose function is muscular coordination. Therefore, heavy indulgence in alcohol is likely to damage it leading to loss of muscular coordination. The improper functioning of the cerebellum causes a drunkard's staggering. Increased drinking might permanently destroy the functioning of the cerebellum. Peripheral neuropathy manifested through numbness and weaknesses in the alcoholic's hands and feet is likely to occur due to the malfunctioning or damaged cerebellum. Alcoholics are also at a higher risk of destroying vulnerable nerves in the arm while sleeping resulting in partial palsy unable to stretch the arm for a longer period.

Specialized neuropsychological tests also prove subtle and mild damage to the brain of alcoholics. Emotional impaired perception, learning challenges, memory loss, and personality changes are detectable in most alcoholics. These changes indicate damage to the brain system by alcohol. The most common is in drunk driving where drunk drivers are incapable of making basic judgments and reading road signs. As a result, they end up causing accidents. The Korsakoff's syndrome (KS) is a severe alcohol-induced mental issue where the individual forgets events as soon as they occur. Individuals with KS have a short-term memory but keep the long-term memory [9]. Consequently, they tend to live in the past. Alcoholics also develop poor attention to events happening around them. As a result, they process visual information slower than non-alcoholics.

\section{Conclusion}

The findings in this paper indicate that alcohol has a direct and indirect effect on the brain and the nervous system. Therefore, it poses a danger to the lives and wellbeing of those who indulge in it. The various parts of the brain control a variety of functions in the body. As a result, indulging in alcohol puts the entire body at risk. Cunningham, et al. [10] established that alcoholism contributed to stroke mortality. In their study, they found out that black and white individuals aged over 45 years old who indulged in heavy consumption of alcohol were at a higher risk of stroke. The average age for a first stroke attack among drinkers was 64.7 years old. The chances for hemorrhage stroke was higher among alcoholics than non-drinkers [10]. Studies of this nature are significant since they help in policy implementation and substance control. They also provide insights into the areas of examination and treatment once alcoholism is identified in a patient. Policy makers and implementing bodies can also rely on these studies to control or restrict the use of alcohol. Intervention measures cut across all affected groups regardless of their age or gender.

\section{References}

1. Costardi J, Nampo R, Silva G, Ribeiro M, Stella H, et al. (2015) A review on alcohol: from the central action mechanism to chemical dependency. Rev Assoc Med Bras 61: 381-387.

2. Erdozain AM, Morentin B, Bedford L, King E, Tooth D, et al. (2014) Alcohol-related brain damage in humans. PLoS One 9: e93586. [Crossref]

3. Sinha R (2017) Chronic alcohol-related neural and peripheral stress system dysfunction: Effects on alcohol craving, relapse and recovery. Alcohol 60: 212.

4. Welch KA (2017) Alcohol consumption and brain health. BMJ 357: j2645. [Crossref]

5. Yang H, Spence J, Briggs R, Rao U, North C, et al. (2014) Interaction between early life stress and alcohol dependence on neural stress reactivity. Addict Biol 20: 523-533.

6. Oscar-Berman M, Marinkovic K (2007) Alcohol: Effects on Neurobehavioral Functions and the Brain. Neuropsychol Rev 17(3), 239-257.

7. Brower K (2001) Alcohol's Effects on Sleep in Alcoholics. Curr Addict Rep 25: 110-122.

8. Roehrs T, Roth T (2001) Sleep, sleepiness, and alcohol use. Alcohol Res Health 25: 101-109. [Crossref]

9. Kabai P, Sabia S, Singh-Manoux A, Kivimaki M, Shipley M, et al. (2014) Alcohol consumption and cognitive decline in early old age. Neurology 83: 476-476.

10. Cunningham S, Mosher A, Judd S, Matz L, Kabagambe E, et al. (2017) Alcohol Consumption and Incident Stroke Among Older Adults. J Gerontol B Psychol Sci Soc Sci 73: 636-648.

Copyright: (C2018 Griffith C. This is an open-access article distributed under the terms of the Creative Commons Attribution License, which permits unrestricted use, distribution, and reproduction in any medium, provided the original author and source are credited. 